\title{
Integração de Tecnologias para Construção de Objetos de Aprendizagem - $O$ case E2D Ensino de Derivadas a Distância
}

\author{
Érico Marcelo Hoff do Amaral ${ }^{12}$, Thaísa Jacintho Müller ${ }^{134}$ \\ ${ }^{1}$ Programa de Pós-Graduação em Informática na Educação - Universidade Federal do \\ Rio Grande do Sul - Porto Alegre, RS - Brasil \\ ${ }^{2}$ Engenharia de Computação - Universidade Federal do Pampa (Unipampa) \\ Bagé, RS - Brasil \\ ${ }^{3}$ Departamento de Matemática - Pontifícia Universidade Católica do Rio Grande do Sul \\ (PUCRS) - Porto Alegre, RS - Brasil \\ ${ }^{4}$ Centro de Ciências Exatas e Tecnológicas - Universidade do Vale do Rio dos Sinos \\ (UNISINOS) - São Leopoldo, RS - Brasil \\ erico.amaral@unipampa.tche.br, thaisa.muller@pucrs.br
}

\begin{abstract}
Resumo. Este artigo apresenta o projeto para integração de um conjunto de tecnologias de softwares, livres e proprietários, elencados para construção de um objeto de aprendizagem voltado para o ensino do tema Derivadas, denominado $E_{2} D$ (Ensino de Derivadas a Distância). Esta é uma proposta para a extensão do projeto denominado "Desenvolvendo Utilitários com GeoGebra”, criado e implementado em um sitio WEB no ano de 2011. O objetivo principal desta pesquisa é promover um maior entendimento do tema Derivadas, por meio da integração de diferentes ferramentas, organizadas segundo critérios fundamentados por diferentes autores das áreas de matemática e informática na educação.
\end{abstract}

Palavras-chave: Derivadas. Ensino a Distância. GeoGebra. Objetos de Aprendizagem.

\section{Integration of Technologies for Building of Learning Objects - The case $\mathrm{E}_{2} \mathrm{D}$ The teaching of Derivates through virtual classes}

\begin{abstract}
This paper presents the project for integration of a group of software technologies, free and paid, listed in order to build a learning object focused on the Teaching of the topic Derivates, called E2D (Teaching Derivates through Virtual Classes). This is a proposal to the extension of the project called "Developing Utilities with GeoGebra", created and implemented in a website in 2011. The main goal of this research is to promote a better understanding about the topic Derivates, through the integration of a group of authorship tools organized according to a standard grounded by different authors of Mathematics and Informatics in education.
\end{abstract}

Keywords: Derivates. Teaching through virtual classes. GeoGebra. Learning Objects. 


\section{Introdução}

É sabido que, ao longo dos anos, as disciplinas matemáticas vêm se caracterizando como aquelas que mais reprovam e causam maior número de evasões em cursos superiores, nas mais variadas instituições. As disciplinas de Cálculo fazem parte deste contexto, de modo que muitas pesquisas vêm sendo realizadas sobre as dificuldades dos alunos, sob os mais variados enfoques. Como exemplo, temos as investigações baseadas em análise de erros, realizadas na Pontifícia Universidade Católica do Rio Grande do Sul desde 1990. (CURY, 1990; CURY; CASSOL, 2004; CURY; MÜLLER, 2004).

Dentre os conteúdos de Cálculo Diferencial e Integral, destaca-se aqui a Derivada, uma vez que é sabido ser de difícil assimilação e, por outro lado, de extrema importância, uma vez que permite estudar, por exemplo, a taxa segundo a qual uma quantidade varia em relação à outra. Além disso, possui diversas aplicações, tais como velocidade, aceleração e outros fenômenos físicos. Os maiores problemas no estudo das Derivadas estão na falta de entendimento do seu conceito, além de problemas pontuais no uso de algumas regras de derivação. Acredita-se que, a partir do uso sincronizado de um conjunto de objetos de aprendizagem e de exercícios virtuais em um ambiente online, que sirvam como um guia de estudos baseados no devido feedback ao aluno em cada situação apresentada, é possível auxiliá-lo na compreensão do tema.

Sendo assim, o objetivo deste trabalho é apresentar o projeto e desenvolvimento de um objeto de aprendizagem que visa promover uma maior aproximação do aluno com o conceito de Derivadas, através de uma ferramenta de ensino a distância com um alto nível de interatividade. Ainda que a intenção seja de fomentar a aprendizagem autônoma, este objeto também poderá ser utilizado como uma aplicação de apoio em atividades presenciais, a fim de amenizar as dificuldades dos alunos e, por sua vez, auxiliar o docente na sua tarefa de transmitir a informação.

A ideia para o desenvolvimento deste material surgiu da experiência realizada de compartilhamento de objetos educacionais criados com o software de Geometria Dinâmica ${ }^{1}$ GeoGebra. (LIEBAN; MÜLLER, 2012) Para tal, foi criado o site denominado "Desenvolvendo Utilitários com GeoGebra", que pode ser acessado em https://sites.google.com/site/geogebrando/.

Como aporte científico para a elaboração do objeto proposto, partiu-se da investigação de modelos eficientes de aprendizagem, como a teoria da carga cognitiva de Sweller (2003), ciclo de aprendizagem de Kolb (1986), teoria de aprendizagem multimídia de Mayer (2001), entre outros. A implementação deste está baseada em um conjunto de ferramentas de autoria.

Para a descrição desta pesquisa, o artigo está estruturado da seguinte forma: na seção 2. é apresentado o referencial teórico sobre o tema; na seção 3. é descrito o projeto, as ferramentas adotadas e a integração com o ambiente "Desenvolvendo Utilitários com GeoGebra" e, finalizando; a seção 4 discorre sobre as conclusões e os resultados iniciais da pesquisa.

\section{Referencial Teórico}

Para o desenvolvimento da proposta aqui apresentada, buscaram-se algumas referências que direcionassem o trabalho de pesquisa no sentido de produzir um material que

${ }^{1}$ Para Gravina et. al. (2011) um software de Geometria Dinâmica é "uma mídia digital que disponibiliza régua e compasso virtuais, que são instrumentos clássicos com os quais são feitas as construções geométricas, só que agora em ambiente virtual". (p.26) 
realmente possa contribuir para um melhor aprendizado de Derivadas a distância, vindo ao encontro de resultados obtidos em pesquisas já realizadas na área. Por fim, é destacado o projeto "Desenvolvendo Utilitários com GeoGebra" (DUG), que serviu como ponto de partida para esta pesquisa.

\subsection{A Matemática e o Ensino a Distância}

Muitas são as discussões acerca da aprendizagem de Matemática quando se fala em Ensino a Distância. Seria esta uma disciplina que não se encaixa em tal modalidade de ensino?

$\mathrm{Na}$ verdade, assim como em qualquer situação de Ensino à Distância $(\mathrm{EaD}), \mathrm{o}$ que se busca é a interação entre os alunos e deste com o professor, bem como o desenvolvimento de uma aprendizagem autônoma, a qual poderá ser baseada em materiais interativos. Segundo Maia e Mattar (2007) "As novas tecnologias geram, sem dúvida, maior interação de professores e alunos, e mesmo entre os próprios alunos, possibilitando justamente a combinação da flexibilidade da interação humana com a independência no tempo e no espaço." (p.8)

Mais especificamente, com relação à aprendizagem de Matemática, Borba, Malheiros e Zulatto (2008) ressaltam: "Quando o foco é a aprendizagem matemática, a interação é uma condição necessária no seu processo. Trocar ideias, compartilhar as soluções encontradas para um problema proposto, expor o raciocínio, são ações que constituem o 'fazer' Matemática”. (p. 27)

Neste sentido, pode-se pensar também na interação entre professores, alunos e tecnologia, que deve estar baseada na experimentação para formulação de conjecturas e testagem de hipóteses. Além disso, "A experimentação se torna algo fundamental, invertendo a ordem de exposição oral da teoria, exemplos e exercícios bastante usuais no ensino tradicional, e permitindo uma nova ordem: investigação e, então, a teorização." (Borba e Penteado, 2007, p. 41) É passível de se considerar que esta experimentação é um aspecto essencial na construção do conhecimento matemático, e é por este motivo que defende-se o uso do software GeoGebra. Ainda de acordo com Borba e Penteado (2007):

\footnotetext{
O enfoque experimental explora ao máximo as possibilidades de rápido feedback das mídias informáticas e a facilidade de geração de inúmeros gráficos, tabelas e expressões algébricas. Por outro lado, essa prática pedagógica estimula a utilização de problemas abertos, de formulação de conjecturas em que a sistematização só se dá como coroamento de um processo de investigação por parte de estudantes (e, muitas vezes, do próprio professor). (p.41)
}

Porém, para que tudo isto aconteça, é necessário estar atento a como utilizar, em ambientes virtuais, uma linguagem matemática adequada. Segundo Behar e Notare (2009), a aprendizagem em Matemática passa essencialmente pela comunicação, a qual envolve, entre outros aspectos, a utilização de gestos, esboços de gráficos e diagramas, bem como a comunicação escrita. Estas autoras ainda destacam a necessidade de um ambiente de criação e da utilização da tela do computador como um "papel virtual". Aliando isto à ideia de que é fundamental que o aluno receba o devido feedback a cada experimentação é que propomos o desenvolvimento de um material que sirva como um guia de estudos interativo. 


\title{
2.2.0 Ensino de Cálculo
}

Historicamente temos observado uma incidência muito grande de reprovações e dificuldades dos alunos em disciplinas de Cálculo Diferencial e Integral. Já é sabido que a maioria destas dificuldades encontra-se em conteúdos de Matemática Básica, que são pré-requisitos para o estudo de limites e derivadas, por exemplo. Mas também acreditamos que os conteúdos específicos de Cálculo devem ser explorados de maneira a proporcionar no aluno a construção do conhecimento de forma mais significativa, amenizando as dificuldades específicas destes tópicos. Analisando as disciplinas de Cálculo de um modo geral, Guimarães (2002) ressalta que:

\begin{abstract}
A disciplina é apresentada em muitas instituições de ensino sob a forma clássica, nunca fugindo do modelo dado por definições, propriedades, exercícios puramente algébricos, aplicações "fechadas" ou poucas aplicações dos conceitos matemáticos ligados ao cotidiano ou à realidade profissional do aluno e com abordagens isoladas, visto que, ou se adota um método gráfico, ou um método algébrico, ou uma abordagem numérica, mas raramente ocorrem as três abordagens de maneira simultânea.
\end{abstract}

Com a utilização de ambientes informatizados apoiados em softwares de Geometria Dinâmica podemos dar mais significado a alguns conceitos de Cálculo. Porém, ainda observa-se que esta não é uma prática muito difundida. Ainda segundo Guimarães (2002), "O processo de inserção dos ambientes informatizados em relação ao Cálculo é de dificuldade acentuada, visto que os professores não criam situações onde o Cálculo poderia ser conceituado em tais ambientes. Não é apenas uma questão de fazer e sim como fazer."

Uma experiência neste sentido pode ser encontrada, por exemplo, em Castro e Melo (2003), que apresentam em seu trabalho uma proposta de estudo de limites através do software Graphmatica. Estes autores destacam que:

\footnotetext{
É possível, no ensino de limites, usar o Graphmatica (1999) para o traçado do gráfico da função a ser calculado o limite e analisá-lo com relação à tendência; permitindo ao aluno que não possui muita habilidade na construção de gráficos, entender o que seja tendência do valor da função quando " $x$ " tende a um certo valor qualquer.
}

Pode-se perceber, neste estudo, indícios de que é possível desenvolver um material que realmente apoie o aluno em seu aprendizado de Cálculo. Sendo assim, pretende-se contribuir para que esta prática seja difundida cada vez mais e que a tecnologia disponível seja usada em favor da aprendizagem da Matemática também em nível superior.

\subsection{Desenvolvendo Utilitários com GeoGebra (DUG)}

Dentro da perspectiva de utilizar recursos tecnológicos, de modo especial softwares de Geometria Dinâmica, foi criado, no ano de 2011, o projeto denominado "Desenvolvendo Utilitários com GeoGebra". A proposta original era de criar uma forma de compartilhar materiais já existentes e utilizados como materiais de aula, ou até mesmo produzidos por alunos. Este compartilhamento atenderia essencialmente 


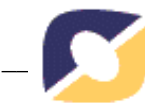

professores interessados em aprimorar suas práticas. Além disso, um dos grandes objetivos do trabalho era incentivar estes professores a fazerem suas próprias criações, inovando suas aulas de acordo com suas necessidades.

Pensando neste apoio a professores foi oferecido, nos meses de setembro e outubro de 2011, um curso de extensão, realizado na Universidade do Vale do Rio dos Sinos. Participaram deste curso alunos de Licenciatura em Matemática e professores da Educação Básica. Paralelamente a isto, foi criado um website, de mesmo nome, onde estão armazenados os materiais utilizados no curso e outros arquivos sobre assuntos diversos. Há também a seção chamada "trabalhos de alunos", na qual são disponibilizadas produções que se destacaram no referido curso ou em outras atividades semelhantes.

A análise desta proposta não se deteve apenas na visão de formação de professores, mas também no apoio ao aprendizado do aluno de um modo geral, uma vez que ele pode, também, ter acesso ao conteúdo do site e interagir com os objetos contidos neste ambiente.

\begin{abstract}
A partir desta experiência e dos estudos apresentados, defendemos que, através de atividades com o GeoGebra, podemos criar um ambiente mais propício para a aprendizagem de Matemática. De fato, com o apoio do software, o aluno percebe a preservação, ou não, de certas propriedades, o que acaba por estimular sua capacidade em conjecturar e estabelecer relações para, então, construir o processo dedutivo, tão importante não só em geometria como em outras áreas da Matemática. (Lieban e Müller, 2012)
\end{abstract}

Por outro lado, a opção por compartilhar com professores e demais interessados atividades apoiadas na tecnologia, difundindo o uso do software, mostrou que tal prática deve ser incentivada, pois oportuniza momentos concretos de percepção e consequentemente de aprendizagem. (LIEBAN; MÜLLER, 2012)

Pensando nisto é que resolveu-se, então, aprimorar o material já iniciado sobre derivadas, na tentativa de promover uma maior aproximação do aluno com o conteúdo, auxiliando, indiretamente, na diminuição das taxas de reprovação em Cálculo.

\title{
3. Metodologia e Projeto do E2D
}

Nesta seção serão apresentados os conceitos envolvidos no projeto e implementação do $\mathrm{E}_{2} \mathrm{D}$, bem como as tecnologias elencadas como suporte ao desenvolvimento da solução.

\subsection{Conceitos para o desenvolvimento de um Objeto de Aprendizagem para o Ensino de Derivadas}

Atualmente é factível afirmar que a Internet, por meio de todas as suas formas de interação, tornou-se um grande repositório virtual, onde é possível minerar-se qualquer tipo de informação multidisciplinar de forma simples e sem a necessidade de expertise no assunto. A educação está inserida dentro deste contexto, sob as mais diferentes formas de exposição via WEB. Lima (2000) afirma que a educação via Internet permite que dados sejam disponibilizados e transformem-se em informação e conhecimento, via um acesso democrático, com alto nível de autonomia, permitindo o suporte educativo a distância, consistindo assim em um processo de interação e suporte ao aprendizado.

Alinhado a esta nova concepção e tendência de ensino, o projeto $E_{2} D$ foi concebido e planejado, vislumbrando disponibilizar, por meio de uma ferramenta WEB de simples utilização, materiais de aprendizagem fundamentalmente estruturados e 
organizados sobre conceitos relacionados ao assunto Derivadas. A fim de que este objeto atenda a um público nativo ou imigrante digital, ou seja, uma plateia exigente e já acostumada com recursos WEB, foram adotados diferentes formatos e tecnologias para a produção do material didático, como animações, vídeos, áudios e hipertexto. Além disto, todos os conceitos apresentados, assim como as atividades propostas, utilizam um vocabulário padrão, com o intuito de se criar uma sintonia fina com as questões matemáticas abordadas, o que segundo Moreira (1983) se torna um elemento facilitador e importante para a ocorrência de uma aprendizagem significativa.

É preciso salientar que o uso de recursos digitais, por si só, não garante uma maneira eficiente de transmissão da informação e conceitos de Derivadas para os alunos. Sendo assim, alguns fundamentos devem ser considerados, tais como a teoria da carga cognitiva de Sweller (2003), a qual descreve como uma das melhores formas de se realizar uma aprendizagem eficiente o alinhamento do processo de informação com o processo cognitivo humano, ou seja, quando o volume de informações oferecidas ao aluno for compatível com a sua capacidade de compreensão. Seguindo esta concepção, $\mathrm{o} \mathrm{E}_{2} \mathrm{D}$ procura seguir um processo linear de apresentação dos seus assuntos, fazendo com que o aluno crie uma base robusta de conhecimentos, os quais são necessários para o desenvolvimento das atividades propostas no ambiente em uma sequência cognitivamente estipulada.

Outro aspecto pertinente a ser considerado no projeto $\mathrm{E}_{2} \mathrm{D}$ está no fato de ter sido implementado com o cuidado de não gerar uma sobrecarga cognitiva no usuário. Partindo deste preceito foram considerados alguns princípios propostos por Mayer (2001), como: o princípio de representação múltipla, com a integração das diferentes tecnologias e mídias; o princípio de proximidade espacial, com os enunciados e rótulos próximos das imagens, vídeos e animações; o princípio das diferenças individuais, permitindo que os alunos de níveis diferentes possam avançar ou recuar dentro do objeto, de acordo com o seu potencial de aprendizagem e conhecimento; o princípio da coerência, com a simplificação dos conteúdos disponibilizados, a fim de que o aluno possa se focar nos assuntos pertinentes; e por último o princípio da redundância, com a integração de áudio, vídeo e animações no mesmo contexto em momentos diferentes de uma atividade especifica no objeto.

A estrutura do $\mathrm{E}_{2} \mathrm{D}$ está apresentada na Figura 01. Seguindo a linha de raciocínio proposta por Ausubel (1968), a fim de alcançar uma aprendizagem significativa, as novas informações disponibilizadas pelo objeto de aprendizagem para Derivadas relacionam-se, de alguma forma, com um elemento relevante da estrutura de conhecimento do indivíduo por meio de uma etapa básica de nivelamento, garantindo que todos os dados apresentados tenham sentido para o aluno.

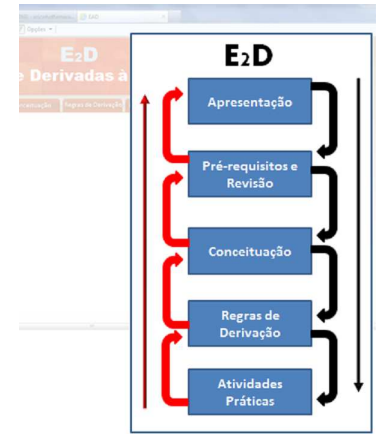

Figura 01 - Estrutura proposta pelo $\mathrm{E}_{2} \mathrm{D}$

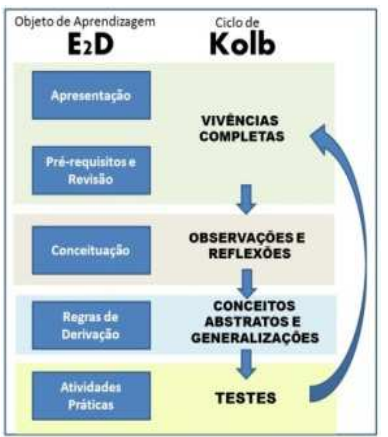

Figura 02- $\mathrm{E}_{2} \mathrm{D}$ e o Ciclo de Kolb 
A organização do objeto segue uma orientação que visa alcançar diferentes níveis de percepção dos alunos. Uma breve descrição dos conteúdos abordados é apresentada abaixo:

1. Na apresentação são destacadas informações relativas ao $\mathrm{E}_{2} \mathrm{D}$, sua composição, uma breve explanação sobre a importância das Derivadas e sua utilidade, sendo findada por uma resenha, de forma multimídia, sobre os principais matemáticos envolvidos nos estudos desta disciplina.

2. Pré-requisitos e Apresentação destacam os conhecimentos prévios necessários para o acompanhamento satisfatório das atividades no ambiente. Esta fase apresenta uma característica interessante, pois disponibiliza uma área de nivelamento ao estudante, com o intuito de que ele consiga construir um conhecimento prévio sobre os assuntos pertinentes ao uso das Derivadas. Considerando os moldes de construção desta ferramenta, é factível dizer que o aluno possui total de liberdade de interagir com a aplicação, podendo utilizar a sua expertise para avançar ou recuar nas etapas disponibilizadas. A tarefa de definir o nível de conhecimento do usuário sobre o tema pode se tornar um fator limitador de um eficiente desenvolvimento do estudante. Ainda é disponibilizado nesta etapa um teste básico de conhecimento, que vislumbra auxiliar o aluno a se situar dentro da organização das atividades, podendo interagir de forma eficaz com o sistema.

3. A fase de Introdução disponibiliza um conjunto de ferramentas interativas, como o GeoGebra, para mostrar a aplicação da Derivada de uma função. Neste contexto os recursos de animação disponíveis são explorados de forma a criar um ambiente didático e interativo para a apresentação dos conceitos básicos de Derivadas, destacando as possibilidades de experimentação garantidas ao aluno.

4. A Conceituação é caracterizada por ser uma importante etapa no conhecimento de Derivadas, visto que nesta fase o estudante terá a possibilidade de reconhecer os princípios primordiais para o entendimento do tema, dentro do contexto estudado. Para ater o aluno neste momento do objeto serão adotados recursos midiáticos, como SlideShows, animações e vídeos (YouTube.com), a fim de prender a atenção dos estudantes e também tornar atrativa a atividade de estudo.

5. A etapa Regras de Derivação utiliza um conjunto de exemplos gráficos e ilustrativos, previamente avaliados e testados, com o intuito de mostrar ao estudante como podem ser usadas tais regras para representação de diferentes fenômenos e suas implicações. Seguindo o padrão já intrínseco do objeto, o aluno possui a disponibilidade de transição de avanço ou recuo de acordo com sua capacidade cognitiva de assimilação.

6. Como última etapa, esta ferramenta proporciona variados exercícios, visando alcançar um alto nível de interação ambiente-aluno. Todos os objetos inseridos neste ponto serão dotados de uma função tutora para a realização das atividades propostas, além de um feedback instantâneo. A organização desta fase está voltada para atender as expectativas e potencialidades identificadas nos participantes, de acordo com o perfil registrado em atividades anteriores. $\mathrm{O}$ estímulo à capacidade de raciocínio e instintos do estudante será parte integrante em todos os momentos e recursos dispostos aqui.

A organização dos objetos e sua disposição no contexto do $\mathrm{E}_{2} \mathrm{D}$, conforme descrito, respeitam o ciclo de aprendizagem apresentado por Kolb (1986), que descreve o processo de concepção da aprendizagem humana em quatro etapas consecutivas, discriminadas na Figura 02, onde: 
- As vivências concretas estão relacionadas com as etapas de Apresentação, Prérequisitos e Revisão do $\mathrm{E}_{2} \mathrm{D}$;

- Observações e Reflexões são partes integrantes da etapa de Conceituação;

- Os conceitos abstratos e generalizações são identificados na etapa Regras de Derivação, permitindo um contato com as diferentes realidades de aplicação do Cálculo;

- Por último têm-se os testes, representados pela etapa Atividades Práticas, na qual é fornecida uma experimentação ativa ao estudante, permitindo o contato com novas situações e resultados, que o remetem novamente ao início do ciclo, a fim de ter acesso a novas vivências concretas e assim consecutivamente.

\subsection{Tecnologias adotadas e a Integração do E2D com o DUG}

Partindo do principio que o $E_{2} D$ é uma aplicação que será integrada ao projeto "Desenvolvendo utilitários com o GeoGebra" dois pontos relevantes foram considerados para sua construção: o primeiro diz respeito à compatibilidade, pois o DUG roda utilizando recursos do Google Apps como servidor de hospedagem e plataforma de desenvolvimento WEB. O segundo ponto está relacionado com o conjunto de objetos que constituirão a ferramenta para o ensino de Derivadas, os quais deverão rodar na internet de forma satisfatória, tendo em vista as diferentes condições de conexão, largura de banda e desempenho dos acessos ao ambiente.

A seguir estão descritas as ferramentas utilizadas para o projeto e criação do $\mathrm{E}_{2} \mathrm{D}$ e de seus objetos de aprendizagem:

a. Para permitir a integração do $\mathrm{E}_{2} \mathrm{D}$ ao DUG optou-se pela adoção de um ambiente de desenvolvimento simples, baseado na linguagem de marcação de hipertexto (HTML), na versão 4, para a definição do template usado, e na versão 5 para a apresentação de áudios e vídeos devido ao seu suporte para as mais recentes multimídias. Foi usado também o eXelearning, uma ferramenta de autoria e código aberto, utilizada para o desenvolvimento de objetos de aprendizagem do tipo rapid e-learning (criação ágil) em HTML, adequada para a produção de conteúdos para e-learning.

b. A ferramenta Hot Potatoes foi essencial para compor exercícios do tipo interativo de escolha múltipla, resposta curta, palavras cruzadas e correspondência, todas estas sob a forma de objetos digitais para publicação na WEB. O resultado desta aplicação está inserido em diferentes partes do $\mathrm{E}_{2} \mathrm{D}$, principalmente na etapa de Atividades Práticas.

c. Com o intuito de criar animações adotou-se recursos do Macromedia Flash, software proprietário que utiliza gráficos, imagens e vídeos para a criação de objetos interativos, que rodam em navegadores, computadores locais e dispositivos móveis. Outra ferramenta adotada para a criação e manipulação de animações e imagens foi o GIMP (GNU Image Manipulation Program), uma aplicação de código aberto focado na disponibilização de recursos eficientes para usuário final. Também para edição de imagens e trabalhos mais pontuais na área de resolução e dimensionamento de figuras e objetos utilizou-se o Corel Draw, um programa de desenho vetorial bidimensional para design gráfico, o qual permitiu a elaboração de representações em alta qualidade.

d. Por último é apresentada a aplicação base para este projeto, o GeoGebra. Este software permite a publicação das tarefas interativas em ambiente WEB de 
forma descomplicada, fato que tornou o DUG atrativo para os alunos. Pensando nisto, todas as etapas do $\mathrm{E}_{2} \mathrm{D}$ estão contempladas com objetos desenvolvidos em GeoGebra.

A Figura 03 mostra a utilização do GeoGebra, por meio do $\mathrm{E}_{2} \mathrm{D}$, onde é disponibilizada uma atividade sobre o uso de derivadas, que permite ao aluno, através da movimentação do ponto $\mathrm{D}$, observar o que acontece quando a reta secante se aproxima da tangente, ao mesmo tempo em que pode ver na tela o cálculo do coeficiente angular de cada reta. Com isso, o estudante percebe que, quanto mais a reta secante se aproxima da reta tangente, mais este coeficiente se aproxima da derivada da função no ponto considerado (abordagem geométrica do conceito de derivada). Já na Figura 04 é apresentado um objeto, também criado com GeoGebra, onde é descrita uma situação simples que ilustra a variação da altura do balão em relação ao tempo, demonstrando desta forma a ideia intuitiva de Derivadas. Quanto menor a variação do tempo, mais próximo se estará da derivada da função no ponto considerado.

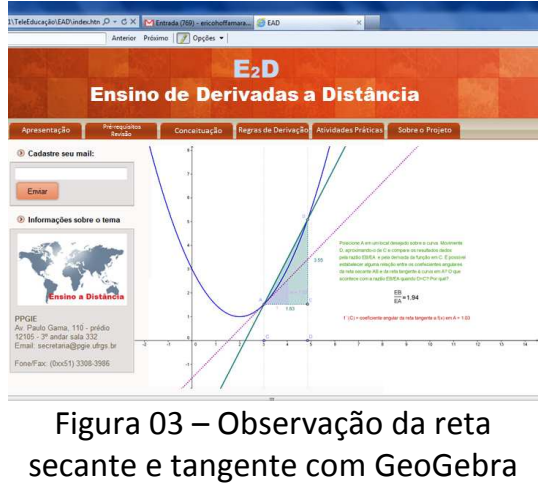

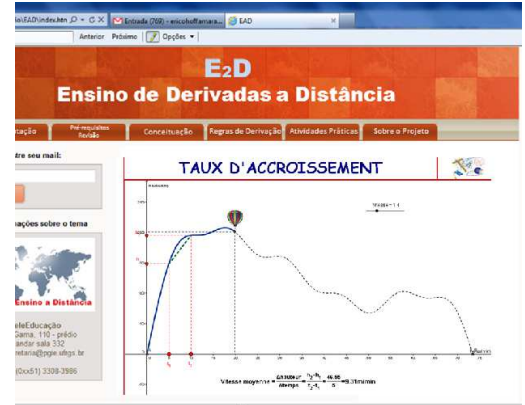

Figura 04 - Variação de altura em relação ao tempo com GeoGebra

Todas as aplicações apresentadas nesta seção estão disponibilizadas no $\mathrm{E}_{2} \mathrm{D}$ de forma relacional, de acordo com os princípios descritos na seção 3.1. A definição dos layouts dos objetos busca alcançar um design de fácil compreensão, a fim de garantir que os estudantes consigam construir o conhecimento do tema de forma significativa.

\section{Resultados e Conclusões}

Sobre o desenvolvimento da ferramenta descrita neste trabalho, pode-se, até o presente momento, afirmar que esta segue padrões testados e que comprovadamente servem como promotores da aprendizagem, uma vez que sua construção foi baseada nos pressupostos apresentados ao longo das seções anteriores. A ferramenta $\mathrm{E}_{2} \mathrm{D}$ é focada na aprendizagem via computador, seguindo a linha do projeto "Desenvolvendo Utilitários com GeoGebra", sobre o qual já é sabido e demonstrado que tem contribuído com alunos e professores de disciplinas matemáticas no apoio a seus estudos, tarefas ou desenvolvimento de suas aulas. Porém, agora o foco encontra-se voltado a questões relativas à aprendizagem de maneira significativa de um conteúdo específico (Derivadas) a distância, com alunos de Cálculo, indo além da proposta inicial de compartilhamento de materiais.

Como se pode observar na revisão bibliográfica que deu suporte a este projeto, a aprendizagem de Cálculo tem sido objeto de estudos e pesquisas, uma vez que é comprovada a grande dificuldade dos alunos nesta disciplina. Espera-se que a 
ferramenta $E_{2} D$ sirva para colaborar com a inserção de ambientes informatizados no ensino deste tema, pois isto ainda tem acontecido de maneira pouco expressiva.

No momento, conclui-se que a integração das diferentes tecnologias de softwares atuais, organizadas de acordo com preceitos de importantes pesquisadores da área do ensino mediado por computador, permite construir um ambiente efetivo para o ensino de Derivadas, de forma presencial e a distância. Como trabalhos futuros pretende-se validar esta aplicação, utilizando como laboratório turmas de Cálculo das Universidades nas quais se tem acesso. $\mathrm{O}$ intuito será avaliar a utilização do $\mathrm{E}_{2} \mathrm{D}$ e identificar até que ponto ele pode auxiliar o aluno em suas dificuldades com o estudo de Derivadas.

\section{Referências}

AUSUBEL, D.P. Educational Psychology: A Cognitive View. New York, Holt, Rinehart and Winston, 1968.

BEHAR, P.A., NOTARE, M. R. A comunicação matemática on-line por meio do ROODA Exata. In: BEHAR, P. A. (Org.). Modelos Pedagógicos em Educação a Distância. Porto Alegre: Artmed, 2009. p. 179-203.

BORBA, M.C.;MALHEIROS, A. P. S; ZULATTO, R. B. A.Educação a Distância online. Belo Horizonte: Autêntica, 2008.

BORBA, M. de C., PENTEADO, M.G. Informática e Educação Matemática. Belo Horizonte: Autêntica, 2007.

CASTRO, A. A. M.; MELO, S. F. Uma proposta pedagógica no ensino do Cálculo Diferencial e Integral I. In: $14^{\circ}$ Congresso de Leitura do Brasil. Campinas, 2003. Anais... Disponível em: http://alb.com.br/arquivomorto/edicoes anteriores/anais14/Sem04/C04003.doc Acesso em 25 abr. de 2012

CURY, H.N. Erros em soluções de problemas de cálculo diferencial e integral: análise, classificação e tentativas de superação. Porto Alegre, PUCRS, Instituto de Matemática, 1990. Relatório de pesquisa. Texto digitado.

CURY, H. N.; CASSOL, M. Análise de erros em Cálculo: uma pesquisa para embasar mudanças. Acta Scientiae, Canoas, v.6, n.1, p. 27-36, jan./ jun. 2004.

CURY, H. N.; MÜLlER, T. J. Análise de erros em matemática em uma visão interdisciplinar. In: CONGRESSO BRASILEIRO DE MATEMÁTICA APLICADA E COMPUTACIONAL, 27., 2004, Porto Alegre. Anais...Porto Alegre; SBAMC, 2004. CD-ROM.

GRAVINA, M.A., et. al. Geometria Dinâmica na Escola. In: GRAVINA, M.A. et. al. (Orgs). Matemática, Mídias Digitais e Didática - tripé para a formação de professores de Matemática. Editora da UFRGS, 2011, p. 26-45.

GUIMARÃES, O. L. C. Cálculo Diferencial e Integral: do Algebrismo às Representações Múltiplas. In: 25a ANPEd -2002 - Caxambu - Minas Gerais. Anais... Disponível em: http://www.anped.org.br/reunioes/25/tp251.htm\#gt19. Acesso em 25 abr. de 2012

KOLB, D; RUBLIN, I; MCINTYRE, J. PsicologiaOrganizacional - uma abordagem vivencial.São Paulo: Ed. Atlas, 1986.

LIEBAN, D. E.; MÜLLER, T. J. Construção de Utilitários com o Software GeoGebra: uma proposta de divulgação da geometria dinâmica entre professores e alunos. Revista do Instituto GeoGebra Internacional de São Paulo, São Paulo, v.1, n.1, p. 37-50, 2012.

LIMA, F. O. A Sociedade Digital. Rio de Janeiro: Qualitymark, 2000. 152p. 
MAIA, C.; MATTAR, J. ABC da EaD: A educação a distância hoje. São Paulo: Pearson Prentice Hall, 2007.

MAYER, R. Multimedia Learning. Cambridge: Cambridge University Press. 2001.

MOREIRA, M. A. Ensino e Aprendizagem - EnfoquesTeóricos. São Paulo: Moraes, 3 edição, 1983.

SWELLER, John. Cognitive Load Theory: A Special Issue of educational Psychologist. LEA, Inc, 2003. 